\title{
SPACE-TIME-DOMAIN DECOMPOSITION FOR OPTIMAL CONTROL PROBLEMS GOVERNED BY LINEAR HYPERBOLIC SYSTEMS
}

\author{
Günter Leugering*
}

\begin{abstract}
In this article, we combine a domain decomposition method in space and time for optimal control problems with PDE-constraints described in [2] to a simultaneous space-time decomposition applied to optimal control problems for systems of linear hyperbolic equations with distributed control. We thereby extend the recent work [31,32] and answer a long standing open question as to whether the combination of time- and space-domain decomposition for the method under consideration can be put into one single convergent iteration procedure. The algorithm is designed for a semi-elliptic system of equations obtained from the hyperbolic optimality system by the way of reduction to the adjoint state. The focus is on the relation to the classical procedure introduced by P. L. Lions [25] for elliptic problems.
\end{abstract}

Key words: space- and time-domain decomposition, optimal control, linear hyperbolic systems, convergence, a posteriori error estimates.

2010 Mathematics Subject Classification: 49-XX,49M05,49M27,49K20.

Communicated by Dr. V. Borsch

\section{Introduction}

Spatial domain decomposition methods as well as time-domain decomposition for partial differential equations (PDEs) have been a subject of intense research in the past. See e.g., the web site http://www.ddm.org where the biannual conferences since 1987 are listed together with the corresponding downloadable proceedings. Obviously, there is an overwhelming amount of literature that cannot be reflected here by any means. The wealth of articles and even monographs related to the topic, however, shrinks drastically when it comes to the decomposition of optimal control problems for partial differential equations and even more when convergence of the iterative methods is considered on the PDE-level. In optimal control of PDEs, the decomposition of the first order optimality system is the most intuitive approach, as the optimization problem is then replaced by a system of coupled PDEs, the state equation and the adjoint equation. Our particular interest is in such methods which result in a decomposed system which, in turn, can be seen as an optimality system associated with a virtual control problem on a smaller space-time domain. In this sense, one aims at the decomposition of the original

\footnotetext{
${ }^{*}$ FAU Senior Fellow of Applied Mathematics, Department of Data Science, Friedrich-AlexanderUniversität Erlangen-Nürnberg Cauerstr. 11 D-91058 Erlangen, Germany (guenter.leugering@fau.de)

(C) G. Leugering, Space-time-domain decomposition, 2021.
} 
optimal control problem to similar problems on smaller domains that, in turn, can be solved in parallel.

Generally speaking, for time-domain decomposition, given a PDE with time domain $[0, T]$, the idea is to introduce a coarse time discretization of $[0, T]$ into a disjoint union of subintervals $I_{k}:=\left[T_{k}, T_{k+1}\right]$ with $[0, T]=\mathrm{cl} \bigcup_{k=1}^{K}\left(T_{k}, T_{k+1}\right)$ and then to iteratively decouple the PDE such that on each subinterval $I_{k}$, the same PDE is solved together with conditions at the breakpoints $T_{k}$ that couple the states at the current iteration $n+1$ with those at iteration $n$. We may trace back the contributions to the seminal paper [7] by J.L. Lions et al., in which the so-called parareal-scheme has been introduced. In [23,24], the authors further developed the scheme and applied it to quantum control problems. This scheme has then later been identified as a variant of the common multiple-shooting method; see, e. g., [8]. These methods, which consist of a coupling of coarse grain discrete-in-time solutions at the break points with a parallel computation of full (respectively, small grain) solutions on the subintervals, were first developed for the mere simulation of nonlinear PDEs. In the article [4], the authors, for the first time, considered the time-domain decomposition of optimal control problems for the time-dependent Maxwell system. Later, in [3], a broad number of such problems - even combined with a spatial domain decomposition for PDEs on networked domains - have been investigated. We also refer to [11] and [9], where methods related to multiple-shooting have been provided along with applications for the heat equation. During the last decade, there has been an increasing interest in applying time- and space-domain decomposition techniques to optimal control problems; see, e.g., [17-22]. The goal of this article is to extend the timedomain decomposition method of $[2,3]$ to optimal control problems involving linear hyperbolic systems of conservation laws to time-space-decompositions. We are particularly interested in applications that focus on processes on metric graphs or networks. As examples, we will focus on networks of strings or rods. Systems that are related to gas flow in pipe networks, after proper linearization around an equilibrium state, are easily seen to fit into the framework of this article-for the model; see, e.g., $[15,16]$. Such problems on metric graphs, where the edges, which are representative of the spatial domains of the corresponding PDEs, are coupled at the vertices of the graph, can be transcribed into two-point initial boundary value problems with a possibly large number of state variables; see Example 2.1 for further explanation. The issue of existence of solutions to optimal control problems, in particular in the context of possibly nonsmooth, say $L^{2}(0, T)$, boundary controls is not settled for the type of systems considered. For a class of distributed controls in the context of semi-linear systems, where the spatial part is fixed, we refer to [12]. As for the well-posedness of the optimality system, we refer to the work [1] of Brokate, but include the arguments for the linear case-which is under consideration here-in section 3 . We also remark that the iterative timedomain decomposition considered here can be interpreted in a way in the context of the classic Robin-Robin-type Schwarz-method introduced by P. L. Lions [25], which has been interpreted by Glowinski and Le Tallec in [26] as a variant of 
their Uzawa-type saddle-point algorithm. This algorithm, in turn, results from an augmented Lagrangian formulation of the interface problem. Indeed, for the timedomain decomposition, say with two time intervals, one may regard the optimality system as a second-order boundary value problem in space and time. The analogue of the P. L. Lions algorithm with the addition of a damped-Richardson relaxation, see [27], is then related to our method. This will be made more explicit in due course. The article is organized as follows. We begin with the problem statement in Section 1, where we include a detailed discussion of an example of a network of controlled strings or rods, in fact a star graph. In Section 2, we introduce the space-time-domain decomposition method for the overall optimality system into systems on the subdomains. In section 3, we gather some known facts about the well-posedness of the optimality system and provide a result for the resulting reduced system. In Section 4, we discuss the convergence of the iteration. Section 5 contains the development of an a posteriori error estimate for our iteration. Finally, in Section 6, we provide an interpretation of the method and the results in the context of the original optimal control problem, whereas in section 7 , we summarize our results and pose some open problems.

\section{Problem Statement}

Let $y(t, x) \in \mathbb{R}^{d}, t \in[0, T], x \in[0, L]$, denote the state and let

$$
\Lambda=\operatorname{diag}\left(\lambda_{1}, \ldots, \lambda_{m}, \lambda_{m+1}, \ldots, \lambda_{d}\right) \in \mathbb{R}^{d \times d}
$$

with constant numbers

$$
\lambda_{1} \geqslant \lambda_{2} \geqslant \cdots \geq \lambda_{m}>0>\lambda_{m+1} \geqslant \ldots \geqslant \lambda_{d},
$$

represent the physics of the system, taken in characteristic coordinates to make the mathematical description simpler. We use the block-matrix abbreviation

$$
\Lambda=\operatorname{diag}\left(\Lambda^{+}, \Lambda^{-}\right),
$$

with $\Lambda^{+}:=\operatorname{diag}\left(\lambda_{1}, \ldots, \lambda_{m}\right)$ and $\Lambda^{-}:=\operatorname{diag}\left(\lambda_{m+1}, \ldots, \lambda_{d}\right)$. Accordingly, we denote the first $m$ components of the state by $y^{+}$and the remaining $d-m$ components by $y^{-}$such that $y=\left(y^{+}, y^{-}\right)^{\top}$. For the sake of simplicity, we consider here separated boundary conditions. To this end, we introduce a block matrix $K$ as follows

$$
K:=\left[\begin{array}{ll}
K^{00} & K^{01} \\
K^{10} & K^{11}
\end{array}\right],
$$

where

$K^{00}=0 \in \mathbb{R}^{m \times m}, \quad K^{01} \in \mathbb{R}^{m \times(d-m)}, \quad K^{10}=0 \in \mathbb{R}^{(d-m) \times m}, \quad K^{11} \in \mathbb{R}^{(d-m) \times(d-m)}$.

Let $B_{d}$ signifies the input operator for distributed controls $u$. In fact, in these notes, we set $B_{d}=I$. We will comment on this special choice in due course. We 
consider separated two-point boundary value problems for systems of hyperbolic linear equations of the form

$$
\begin{aligned}
\partial y t+\Lambda \partial y x & =B_{d} u, & (t, x) \in(0, T) \times(0, L), \\
\left(\begin{array}{c}
y^{+}(t, 0) \\
y^{-}(t, L)
\end{array}\right) & =K\left(\begin{array}{l}
y^{+}(t, L) \\
y^{-}(t, 0)
\end{array}\right), & t \in(0, T), \\
y(0, x) & =y_{0}(x), & x \in(0, L),
\end{aligned}
$$

Moreover, $u$ is taken to represent distributed controls, where $u(t, x) \in \mathbb{R}^{d}$. Finally, $y_{0}(x) \in \mathbb{R}^{d}$ for $x \in[0, L]$ denotes the initial data. We should note that the boundary conditions in (2.2) are in accordance with the standard formulation as in Chapter 6 of [6]. Under these conventions, system (2.2) is a controlled hyperbolic and linear system. In addition to (2.2), we consider the natural tracking-type cost function

$$
J(u, y):=\frac{\kappa}{2} \int_{0}^{T} \int_{0}^{L}\left\|y-y_{d}\right\|^{2} \mathrm{~d} x \mathrm{~d} t+\frac{\nu}{2} \int_{0}^{T} \int_{0}^{L}\|u\|^{2} \mathrm{~d} x \mathrm{~d} t .
$$

The considered control problem is thus given by

$$
\min _{u, y} J(u, y) \quad \text { s.t. }(u, y) \text { satisfies }(2.2) \text {. }
$$

It is a matter of standard variations to derive the adjoint system from the first variation of the Lagrangian function

$$
L(u, y, p)=J(u, y)+\int_{0}^{T} \int_{0}^{L} p^{\top}\left(\partial y t+\Lambda \partial y x-B_{d} u\right) \mathrm{d} x \mathrm{~d} t .
$$

The details are left to the reader. We obtain the following optimality conditions governing the adjoint variable $p$

$$
\begin{array}{rlrl}
\partial_{t} p+\Lambda \partial_{x} p & =\kappa\left(y-y_{d}\right), & (t, x) \in(0, T) \times(0, L), \\
\left(\begin{array}{c}
p^{+}(t, L) \\
p^{-}(t, 0)
\end{array}\right) & =\tilde{K}\left(\begin{array}{c}
p^{+}(t, 0) \\
p^{-}(t, L)
\end{array}\right), & t \in(0, T), \\
p(T, x) & =0, & x & \in(0, L),
\end{array}
$$

where the boundary matrix $\tilde{K}$ is given by

$$
\tilde{K}:=\operatorname{diag}\left(\left(\Lambda^{+}\right)^{-1},\left|\left(\Lambda^{-}\right)^{-1}\right|\right) K^{\top} \operatorname{diag}\left(\Lambda^{+},\left|\Lambda^{-}\right|\right),
$$

and the absolute value signs indicates absolute values of the diagonal entries.

After solving (2.5), the required distributed controls are computed by invoking the following formula

$$
u(t, x)=\frac{1}{\nu} B_{d}^{\top} p(t, x), \quad(t, x) \in(0, T) \times(0, L),
$$

obtained from the first variation of the Lagrangian function. 
Example 2.1 (A network of strings). We consider a star-graph consisting of $m$ strings or rods connected at a multiple node located at $x=0$. The individual strings are stretched along an interval $[0, L]$. Each string is represented by a displacement $w_{i}(t, x)$ for $x \in[0, L]$ and $t \in[0, \infty)$. We assume, for the sake of simplicity, that the stiffness of the strings $c_{i}$ are constant and are, in fact, even identical to 1 . The more general case can be handled easily but necessitates more detailed description which, in turn, is not appropriate here. These strings or rods form a network located in the plane and $w_{i}(t, x)$ is either the out-of-the-place displacement of the $i$-th string or the longitudinal displacement of the $i$-th rod. We assume that the strings (or rods) satisfy a linear undamped wave equation such that at $x=0$, the displacements are equal for all times and the sum of forces is 0 . At the simple nodes, i. e., at $x=L$, the strings $i=2, \ldots, m$ are subject to Neumann boundary conditions, while string $i=1$ is clamped (i. e., Dirichlet boundary condition). The corresponding system can be written as

$$
\begin{array}{rlrl}
\partial_{t t} w_{i}-\partial_{x x} w_{i} & =\left(B_{d} u\right)_{i}, & (t, x) \in(0, T) \times(0, L), i=1, \ldots, m, \\
w_{i}(t, 0) & =w_{j}(t, 0), & & \text { in }(0, T), \\
\sum_{i=1}^{m} \partial_{x} w_{i}(t, 0) & =0, & & t \in(0, T), i, j=1, \ldots, m, \\
w_{1}(t, L) & =0, & \\
\partial_{x} w_{i}(t, L) & =0, & t \in(0, T), i=2, \ldots, m, \\
w_{i}(0, x) & =w_{i 0}(x), & x \in(0, L), i=1, \ldots, m, \\
\partial_{t} w_{i}(0, x) & =w_{i 1}(x), & x \in(0, L), i=1, \ldots, m .
\end{array}
$$

We now transform (2.8) into the format (2.2). In a first step, we transform (2.8a) into a $2 \times 2$-system. To this end, we set

$$
z_{i, 1}:=\frac{1}{2}\left(\partial_{t} w_{i}-\partial_{x} w_{i}\right), \quad z_{i, 2}:=\frac{1}{2}\left(\partial_{t} w_{i}+\partial_{x} w_{i}\right)
$$

Hence

$$
\partial_{t} w_{i}=z_{i, 1}+z_{i, 2}, \quad \partial_{x} w_{i}=z_{i, 2}-z_{i, 1},
$$

and, therefore

$$
\partial_{t}\left(\begin{array}{c}
z_{i, 1} \\
z_{i, 2}
\end{array}\right)+\left[\begin{array}{cc}
1 & 0 \\
0 & -1
\end{array}\right] \partial_{x}\left(\begin{array}{l}
z_{i, 1} \\
z_{i, 2}
\end{array}\right)=\frac{1}{2}\left(\begin{array}{l}
B_{d} u_{i} \\
B_{d} u_{i}
\end{array}\right) .
$$

We define

$$
y_{i}=z_{i, 1}, \quad i=1, \ldots, m ; \quad y_{i}=z_{i-m, 2}, \quad i=m+1, \ldots, 2 m=: d .
$$

Then, the transmission conditions $(2.8 \mathrm{~b})$ and $(2.8 \mathrm{c})$ can be equivalently written as

$$
\left(\begin{array}{c}
y_{1} \\
\vdots \\
y_{m}
\end{array}\right)(t, 0)=-\frac{1}{m}\left[\begin{array}{cccc}
m-2 & -2 & \cdots & -2 \\
-2 & m-2 & \cdots & -2 \\
\vdots & \vdots & \ddots & \vdots \\
-2 & -2 & \cdots & m-2
\end{array}\right]\left(\begin{array}{c}
y_{m+1} \\
\vdots \\
y_{d}
\end{array}\right)(t, 0)
$$


We introduce the matrix $S$ such that

$$
(S \varphi)_{i}:=\left(\frac{2}{m} \sum_{j=1}^{m} \varphi_{j}-\varphi_{i}\right) .
$$

Thus, (2.9) reads as $y^{+}(0)=S y^{-}(0)$, where we have set $y^{+}:=\left(y_{1}, \ldots, y_{m}\right)^{\top}$ and $y^{-}:=\left(y_{m+1}, \ldots, y_{d}\right)^{\top}$. The matrix $S$ has nice properties. It can be interpreted as a scattering matrix. In particular,

$$
\sum_{i=1}^{m}(S \varphi)_{i}=\sum_{i=1}^{m} \varphi_{i} \quad \text { and } \quad S S \varphi=\varphi
$$

holds. At $x=L$, we have, at least formally for sufficiently regular states,

$$
\partial_{t} w_{1}(t, L)=0 \quad \Rightarrow \quad z_{1,1}(t, L)+z_{1,2}(t, L)=0 \quad \Rightarrow \quad y_{1}(t, L)=-y_{m+1}(t, L)
$$

for the clamped string and

$$
\partial_{x} w_{i}(t, L)=0 \quad \Rightarrow \quad z_{i, 2}(t, L)-z_{i, 1}(t, L)=0
$$

for the other strings. This provides the boundary conditions at the end $x=L$ :

$$
\begin{aligned}
& y_{1}(t, L)=-y_{m+1}(t, L), \\
& y_{i}(t, L)=y_{m+i}(t, L), \quad i=2, \ldots, m .
\end{aligned}
$$

In case of $K^{00}=0, K^{11}=0$, as well as $K^{01}=S$ and $K^{10}=\operatorname{diag}(-1, \ldots,-1)$, we obtain the boundary condition

$$
\left(\begin{array}{c}
y^{+}(t, 0) \\
y^{-}(t, L)
\end{array}\right)=\left[\begin{array}{cc}
0 & K^{01} \\
K^{10} & 0
\end{array}\right]\left(\begin{array}{l}
y^{+}(t, L) \\
y^{-}(t, 0)
\end{array}\right) .
$$

Thus, our example is of the format (2.2), however, with separated boundary conditions.

Remark 2.1. We remark that there are many more examples that exactly fit into this framework after linearization. These are, e.g., networks of open channels with the dynamics governed by the shallow water equations with wall friction (see, e.g., [13]), or networks of gas pipelines [15]. Moreover, networks of linear Timoshenko beams (see the semi-linear model in [14]) can be written in the framework of (2.2) as well. In these notes, we insist on linear systems in order to make the procedure clear. The semi-linear case is treated in a forthcoming publication.

In contrast to [31,32], we eliminate the state variable via formal differentiating the differential equation in (2.5) w.r.t $x$ followed by multiplying by $\Lambda$ and w.r.t $t$. We arrive at

$$
\begin{aligned}
\Lambda \partial_{x t} p+\Lambda^{2} \partial_{x x} p & =\kappa\left(\frac{1}{\nu} B_{d} B_{d}^{\top} p-\partial_{t} y-\partial_{x} y_{d}\right), \\
\partial_{t t} p+\Lambda \partial_{x t} p & =\kappa\left(\partial_{t} y-\partial_{t} y_{d}\right) .
\end{aligned}
$$


The second equation of (2.10) yields

$$
\kappa \partial_{t} y=\partial_{t t} p+\Lambda \partial_{x t} p+\kappa \partial_{t} y_{d}
$$

which used in the first equation of (2.10) to obtain

$$
\partial_{t t} p+2 \Lambda \partial_{t x} p+\Lambda^{2} \partial_{x x} p-\frac{\kappa}{\nu} B_{d} B_{d}^{\top} p=\kappa\left(\partial_{t} y_{d}+\Lambda \partial_{x} y_{d}\right)=:-\kappa f .
$$

We introduce the block matrix

$$
\mathcal{A}:=\left[\begin{array}{cc}
I & \Lambda \\
\Lambda & \Lambda^{2}
\end{array}\right]
$$

which is symmetric and positive semi-definite. Indeed, with

$$
\Phi=\left(\phi_{1}, \phi_{2}\right)^{\top}, \quad \phi_{1}, \phi_{2} \in \mathbb{R}^{d},
$$

we have

$$
\Phi^{\top} \mathcal{A} \Phi=\left\|\phi_{1}+\Lambda \phi_{2}\right\|^{2}>0
$$

unless $\Phi \in \operatorname{ker} \mathcal{A}=\left\{\Phi: \phi_{1}+\Lambda \phi_{2}=0\right\}$ in which case $\Phi^{\top} \mathcal{A} \Phi=0$. Define the timespace nabla-operator

$$
\nabla:=\left(\partial_{t}, \partial_{x}\right)^{\top}
$$

then with the divergence $\operatorname{div} \Phi:=\nabla \cdot \Phi=\partial_{t} \phi_{1}+\partial_{x} \phi_{2}$ and the gradient $\operatorname{grad} \phi$ $:=\nabla \phi=\left(\partial_{t} \phi, \partial_{x} \phi\right)^{\top}, \phi \in \mathbb{R}^{d}$, (2.11) turns into a degenerate Poisson equation in divergence form

$$
-\nabla \cdot(\mathcal{A} \nabla p)+\frac{\kappa}{\nu} B_{d} B_{d}^{\top} p=\kappa f .
$$

Clearly, equation (2.15) holds on the rectangular domain $\Omega:=(0, T) \times(0, L)$. In order to rewrite the initial and boundary conditions in (2.2), (2.5) in terms of the state $p$ and its co-normal derivative related to $\mathcal{A}$ (see, e. g., [30]) we note that: 1) the outer normal orts at the time-like boundaries $\Gamma_{3}:=\{(0, x) \mid x \in(0, L)\}$ and $\Gamma_{4}:=\{(T, x) \mid x \in(0, L)\}$ are $\left(\nu_{t}, \nu_{x}\right)=(-1,0)$ and $\left(\nu_{t}, \nu_{x}\right)=(+1,0)$, respectively, while the outer normal orts at the space-like boundaries $\Gamma_{1}:=\{(t, 0) \mid t \in(0, T)\}$ and $\Gamma_{2}:=\{(t, L) \mid t \in(0, T)\}$ are $\left(\nu_{t}, \nu_{x}\right)=(0,-1)$ and $\left(\nu_{t}, \nu_{x}\right)=(0,+1)$, respectively; 2$) \mathcal{A} \in \mathbb{R}^{2 d \times 2 d}$, such that each block $\left(a_{i j}\right)_{i, j=1}^{2}$ of $\mathcal{A}$ is in $\mathbb{R}^{d \times d}$. Then, denoting the classical trace of $p$ by $\operatorname{tr}(p)=p(t, x),(t, x) \in \partial \Omega$, and relating the values 1 and 2 of the indices $i, j$ to the directions $t$ and $x$, respectively, we have

$$
\partial_{\nu_{\mathcal{A}}} p(t, x):=\sum_{i, j=1}^{2} a_{i j} \operatorname{tr}\left(\partial_{j} p(t, x)\right) \nu_{i}(t, x), \quad(t, x) \in \partial \Omega .
$$

Using this definition, we can write the co-normal derivatives explicitly as follows

$$
\begin{aligned}
-\partial_{\nu_{\mathcal{A}}} p(t, 0) & =\Lambda\left(\partial_{t} p(t, 0)+\Lambda \partial_{x} p(t, 0)\right), & & t \in(0, T), \\
\partial_{\nu_{\mathcal{A}}} p(t, L) & =\Lambda\left(\partial_{t} p(t, L)+\Lambda \partial_{x} p(t, L)\right), & & t \in(0, T), \\
-\partial_{\nu_{\mathcal{A}}} p(0, x) & =\partial_{t} p(0, x)+\Lambda \partial_{x} p(0, x), & & x \in(0, L), \\
\partial_{\nu_{\mathcal{A}}} p(T, x) & =\partial_{t} p(T, x)+\Lambda \partial_{x} p(T, x), & & x \in(0, L) .
\end{aligned}
$$


We now go back to the boundary conditions at $x \in\{0, L\}$ in $(2.2),(2.5),(2.1)$ which in the special case of this article are rewritten as

$$
\begin{aligned}
& \left(\begin{array}{c}
y^{+}(t, 0) \\
y^{-}(t, L)
\end{array}\right)=\left(\begin{array}{l}
K^{01} y^{-}(t, 0) \\
K^{10} y^{+}(t, L)
\end{array}\right), \quad t \in(0, T), \\
& \left(\begin{array}{c}
p^{+}(t, L) \\
p^{-}(t, 0)
\end{array}\right)=\left(\begin{array}{c}
\left(\Lambda^{+}\right)^{-1}\left(K^{10}\right)^{\top}\left|\left(\Lambda^{-}\right)\right| p^{-}(t, L) \\
\left|\left(\Lambda^{-}\right)\right|^{-1}\left(K^{01}\right)^{\top}\left(\Lambda^{+}\right) p^{+}(t, 0)
\end{array}\right), \quad t \in(0, T),
\end{aligned}
$$

or in short

$$
\begin{aligned}
\left(I,-K^{01}\right) y(t, 0) & =0, & \left(K^{10},-I\right) y(t, L) & =0, \\
\left(\left(K^{01}\right)^{\top}, I\right) \Lambda p(t, 0) & =0, & \left(I,\left(K^{10}\right)^{\top}\right) \Lambda p(t, L) & =0 .
\end{aligned}
$$

We recall the relation between $y$ and $p$

$$
y=\frac{1}{\kappa}\left(\partial_{t} p+\Lambda \partial_{x} p\right)+y_{d}
$$

which, in turn, can be expressed by the co-normal derivative along the boundary as

$$
y(t, x)=\frac{1}{\kappa} \partial_{\nu_{\mathcal{A}}} p(t, x)+y_{d}(t, x), \quad(t, x) \in \partial \Omega .
$$

Equation (2.21) can be used to rewrite (2.20) as follows

$$
\begin{aligned}
\mathcal{B}_{11} \partial_{\nu_{\mathcal{A}}} p(t, 0) & =-\kappa \mathcal{B}_{11} y_{d}(t, 0), & \mathcal{B}_{21} \partial_{\nu_{\mathcal{A}}} p(t, L) & =-\kappa \mathcal{B}_{21} y_{d}(t, L), \\
\mathcal{B}_{10} p(t, 0) & =0, & \mathcal{B}_{20} p(t, L) & =0,
\end{aligned}
$$

where we have denoted

$$
\begin{array}{ll}
\mathcal{B}_{11}:=\left(I,-K^{01}\right), & \mathcal{B}_{21}:=\left(K^{10},-I\right), \\
\mathcal{B}_{10}:=\left(\left(K^{01}\right)^{\top}, I\right) \Lambda, & \mathcal{B}_{20}:=\left(I,\left(K^{10}\right)^{\top}\right) \Lambda .
\end{array}
$$

We observe the orthogonality relations $\mathcal{B}_{11} \mathcal{B}_{10}^{\top}=0, \mathcal{B}_{21} \mathcal{B}_{20}^{\top}=0$. Thus, (2.23) provides full boundary conditions of mixed type at the space-like boundaries $\Gamma_{1}$ and $\Gamma_{2}$, while at the time-like boundaries $\Gamma_{3}$ and $\Gamma_{4}$ we have

$$
p(T, x)=0, \quad \partial_{\nu_{\mathcal{A}}} p(0, x)=\kappa\left(y_{0}(x)-y_{d}(0, x)\right), \quad x \in(0, L),
$$

in fact, we may, for the sake of homogeneous notation, introduce $\mathcal{B}_{40}=I, \mathcal{B}_{41}=0$, $\mathcal{B}_{31}=I, \mathcal{B}_{30}=0$. We put (2.15) together with (2.23) and (2.25) in order to finally formulate a system of semi-elliptic boundary value problems in $\Omega$ :

$$
\begin{aligned}
-\nabla \cdot(\mathcal{A} \nabla p)+\frac{\kappa}{\nu} B_{d} B_{d}^{\top} p & =-\kappa\left(\partial_{t} y_{d}+\Lambda \partial_{x} y_{d}\right):=\kappa f & & (t, x) \in \Omega, \\
\mathcal{B}_{11} \partial_{\nu_{\mathcal{A}}} p(t, x) & =-\kappa \mathcal{B}_{11} y_{d}(t, x), \mathcal{B}_{10} p(t, x)=0, & & (t, x) \in \Gamma_{1}, \\
\mathcal{B}_{21} \partial_{\nu_{\mathcal{A}}} p(t, x) & =-\kappa \mathcal{B}_{21} y_{d}(t, x), \mathcal{B}_{20} p(t, x)=0, & & (t, x) \in \Gamma_{2}, \\
\mathcal{B}_{31} \partial_{\nu_{\mathcal{A}}} p(t, x) & =\kappa\left(y_{0}(x)-y_{d}(t, x)\right), & & (t, x) \in \Gamma_{3}, \\
\mathcal{B}_{40} p(t, x) & =0, & & (t, x) \in \Gamma_{4} .
\end{aligned}
$$


Remark 2.2. It is important to note that the kernel of $\mathcal{A} \in \mathbb{R}^{2 d \times 2 d}$ consists of the elements $\Phi$ (2.13) such that $\phi_{1}+\Lambda \phi_{2}=0$. Therefore, when applied to $\nabla p$, we see that the differential operator in (2.15) vanishes if and only if $p$ satisfies the equation

$$
\partial_{t} p+\Lambda \partial_{x} p=0
$$

but this is impossible unless $y_{0}=y_{d}(0, \cdot)$ and $y_{d}$ satisfies the same equation and the boundary conditions in (2.2). In this case, the target function is a solution of the original problem with the right initial data and boundary conditions and, consequently, the adjoint variable is identical to zero. This is also intuitive, as under these conditions the cost functional is zero without any control.

We are now going to apply a domain decomposition method to (2.26). The method that we apply is taken from the work of P. L. Lions [25] designed for scalar elliptic problem. This method has been extended to elliptic and hyperbolic problems on networked domains in [2]. In the same spirit, very recently, there has been an extension to time-domain decomposition of optimal control problems for systems of first order semi-linear equations [31,32] including boundary controls and constraints. The decomposition of such problems w.r.t spatial domains, in particular the decoupling within the boundary conditions, which, in turn, relates to a substructuring is under way. In [3] time- and spatial-domain decompositions have been considered, however, in a sequential way. It was noted there that the convergence of the joint time- and spatial-domain decomposition remained open. With the current interpretation of the original optimality system for the state and the adjoint variable $y, p$, respectively, we first attempt the mentioned convergence using an analogue of the scheme proposed in [25].

To this end, we introduce a special decomposition of the time-space domain $\Omega$ in 4 pieces. Obviously, the general decomposition can be handled similarly. To be more specific, we introduce w.l.o.g. $2 t_{1}=T, 2 x_{1}=L$ and organize the subdomains counterclockwise as follows

$$
\begin{array}{ll}
\Omega_{1}:=\left\{(t, x) \in\left(0, t_{1}\right) \times\left(0, x_{1}\right)\right\}, & \Omega_{2}:=\left\{(t, x) \in\left(0, t_{1}\right) \times\left(x_{1}, L\right)\right\}, \\
\Omega_{3}:=\left\{(t, x) \in\left(t_{1}, T\right) \times\left(x_{1}, L\right)\right\}, & \Omega_{4}:=\left\{(t, x) \in\left(t_{1}, T\right) \times\left(0, x_{1}\right)\right\} .
\end{array}
$$

We denote the interfaces $\Gamma_{i j}=\Gamma_{j i}$ between to adjacent domains as

$$
\begin{array}{ll}
\Gamma_{12}:=\left\{\left(t, x_{1}\right) \mid t \in\left(0, t_{1}\right)\right\}, & \Gamma_{23}:=\left\{\left(t_{1}, x\right) \mid x \in\left(x_{1}, L\right)\right\}, \\
\Gamma_{34}:=\left\{\left(t, x_{1}\right) \mid t \in\left(t_{1}, T\right)\right\}, & \Gamma_{41}:=\left\{\left(t_{1}, x\right) \mid x \in\left(0, x_{1}\right)\right\} .
\end{array}
$$

With this, we can describe the boundaries of the sub-domains. These consist of the transmission boundaries and the external boundaries: $\Gamma_{1, \text { ext }}:=\left(\Gamma_{1} \cup \Gamma_{3}\right) \cap \bar{\Omega}_{1}$, $\Gamma_{2, e x t}:=\left(\Gamma_{2} \bigcup \Gamma_{3}\right) \bigcap \bar{\Omega}_{2}, \Gamma_{3, e x t}:=\left(\Gamma_{2} \bigcup \Gamma_{4}\right) \bigcap \bar{\Omega}_{3}, \Gamma_{4, e x t}:=\left(\Gamma_{4} \bigcup \Gamma_{1}\right) \bigcap \bar{\Omega}_{4}$,

$$
\begin{aligned}
& \partial \Omega_{1}=\Gamma_{12} \bigcup \Gamma_{14} \bigcup \Gamma_{1, e x t}, \quad \partial \Omega_{2}=\Gamma_{21} \bigcup \Gamma_{23} \bigcup \Gamma_{2, e x t}, \\
& \partial \Omega_{3}=\Gamma_{32} \bigcup \Gamma_{34} \bigcup \Gamma_{3, e x t}, \quad \partial \Omega_{4}=\Gamma_{41} \bigcup \Gamma_{43} \bigcup \Gamma_{4, e x t} .
\end{aligned}
$$


We provide the Green formula on $\Omega$

$$
\begin{aligned}
0 & =\int_{\Omega} \phi^{\top}\left(-\nabla \cdot(\mathcal{A} \nabla p)+\frac{\kappa}{\nu} B_{d} B_{d}^{\top} p-\kappa f\right) d \omega=-\int_{\partial \Omega} \phi^{\top} \partial_{\nu \mathcal{A}} p d \gamma \\
& +\int_{\Omega}(\nabla \phi)^{\top} \mathcal{A} \nabla p d \omega+\frac{\kappa}{\nu} \int_{\Omega} \phi^{\top} B_{d} B_{d}^{\top} p d \omega-\kappa \int_{\Omega} \phi^{\top} f d \omega \\
& =\int_{\Omega}\left(\partial_{t} \phi+\Lambda \partial_{x} \phi\right)^{\top}\left(\partial_{t} p+\Lambda \partial_{x} p\right) d \omega+\frac{\kappa}{\nu} \int_{\Omega}\left(\phi^{\top} B_{d}\right)\left(B_{d}^{\top} p\right) d \omega \\
& +\int_{\Gamma_{1}} \Lambda \phi^{\top}\left(\partial_{t} p+\Lambda \partial_{x} p\right) d \gamma-\int_{\Gamma_{2}} \Lambda \phi^{\top}\left(\partial_{t} p+\Lambda \partial_{x} p\right) d \gamma \\
& +\int_{\Gamma_{3}} \phi^{\top}\left(\partial_{t} p+\Lambda \partial_{x} p\right) d \gamma-\int_{\Gamma_{4}} \phi^{\top}\left(\partial_{t} p+\Lambda \partial_{x} p\right) d \gamma-\kappa \int_{\Omega} \phi^{\top} f d \omega .
\end{aligned}
$$

Let us assume for the sake of simplicity that the target $y_{d}$ is consistent with $y$ in $(2.2)$, i. e.,satisfies the same initial and boundary conditions, while within the domain it is not determined by the state. Then the boundary value contributions in (2.30) (see Remark 2.2) vanish and we obtain

$$
\begin{array}{r}
\int_{\Omega} \phi^{\top}\left(-\nabla \cdot(\mathcal{A} \nabla p)+\frac{\kappa}{\nu} B_{d} B_{d}^{\top} p-\kappa f\right) d \omega=-\kappa \int_{\Omega} \phi^{\top} f d \omega \\
+\int_{\Omega}\left(\partial_{t} \phi+\Lambda \partial_{x} \phi\right)^{\top}\left(\partial_{t} p+\Lambda \partial_{x} p\right) d \omega+\frac{\kappa}{\nu} \int_{\Omega}\left(\phi^{\top} B_{d}\right)\left(B_{d}^{\top} p\right) d \omega .
\end{array}
$$

If we further take $p$ instead of $\phi$ as the test function in (2.31) then we arrive at

$$
\begin{aligned}
& \int_{\Omega} p^{\top}\left(-\nabla \cdot(\mathcal{A} \nabla p)+\frac{\kappa}{\nu} B_{d} B_{d}^{\top} p-\kappa f\right) d \omega \\
= & \int_{\Omega}\left(\partial_{t} p+\Lambda \partial_{x} p\right)^{2} d \omega+\frac{\kappa}{\nu} \int_{\Omega}\left\|B_{d}^{\top} p\right\|^{2} d \omega-\kappa \int_{\Omega} p^{\top} f d \omega .
\end{aligned}
$$

If we we now define $p_{i}:=\left.p\right|_{\Omega_{i}}, i=1, \ldots, 4$, and use the decomposition of $\Omega=\bigcup_{i=1}^{4} \Omega_{i}$ in $(2.30)$, then after taking proper variations in $\phi$, we obtain along the interfaces $\Gamma_{i j}, i, j=1,2,3,4$, the transmission conditions

$$
\begin{array}{ll}
\left.\partial_{\nu_{\mathcal{A}_{1}}} p_{1}\right|_{\Gamma_{12}}+\left.\partial_{\nu_{\mathcal{A}_{2}}} p_{2}\right|_{\Gamma_{21}}=0, & \left.p_{1}\right|_{\Gamma_{12}}=\left.p_{2}\right|_{\Gamma_{21}}, \\
\left.\partial_{\nu_{\mathcal{A}_{1}}} p_{1}\right|_{\Gamma_{14}}+\left.\partial_{\nu_{\mathcal{A}_{4}}} p_{4}\right|_{\Gamma_{41}}=0, & \left.p_{1}\right|_{\Gamma_{14}}=\left.p_{4}\right|_{\Gamma_{41}}, \\
\left.\partial_{\nu_{\mathcal{A}_{2}}} p_{2}\right|_{\Gamma_{23}}+\left.\partial_{\nu_{\mathcal{A}_{3}}} p_{3}\right|_{\Gamma_{32}}=0, & \left.p_{2}\right|_{\Gamma_{23}}=\left.p_{3}\right|_{\Gamma_{32}}, \\
\left.\partial_{\nu_{\mathcal{A}_{3}}} p_{3}\right|_{\Gamma_{34}}+\left.\partial_{\nu_{\mathcal{A}_{4}}} p_{4}\right|_{\Gamma_{43}}=0, & \left.p_{3}\right|_{\Gamma_{34}}=\left.p_{4}\right|_{\Gamma_{43}} .
\end{array}
$$

In order to abbreviate and in order to indicate the generic extension to a more general splitting, we introduce the notation

$$
\Omega:=\bigcup_{i=1}^{N} \Omega_{i}, \quad \mathcal{I}:=\{1, \ldots, N\}, \quad \mathcal{I}_{j}:=\left\{i \in \mathcal{I} \mid \Gamma_{i j}=\bar{\Omega}_{i} \bigcap \bar{\Omega}_{j} \neq \emptyset\right\} .
$$


In the case discussed above $N=4$. The point of the time-space domain decomposition method is now to replace (2.33) by an iteration in order to decouple the transmission across the artificial interfaces $\Gamma_{i j}$. To this end, we introduce the iteration index $n \in \mathbb{N}$ and set

\section{Algorithm DDM}

1. Given $p_{i}^{n}, \partial \nu_{\mathcal{A}_{i}} p_{i}^{n}$ on $\Gamma_{i j}, i \neq j \in \mathcal{I}$.

2. Compute $p_{i}^{n+1}, i \in \mathcal{I}$ according to

$$
\begin{aligned}
& -\nabla \cdot\left(\mathcal{A}_{i} \nabla p_{i}^{n+1}\right)+\frac{\kappa}{\nu} B_{d} B_{d}^{\top} p_{i}^{n+1}=\kappa f_{i}, \quad \text { in } \Omega, \\
& \mathcal{B}_{i 1} \partial_{\nu_{\mathcal{A}_{i}}} p_{i}^{n+1}=0, \quad \mathcal{B}_{i 0} p_{i}^{n+1}=0, \quad \text { on } \Gamma_{i, \text { ext }} \\
& \partial \nu_{\mathcal{A}_{i}} p_{i}^{n+1}+\beta_{i j} p_{i}^{n}=-\partial \nu_{\mathcal{A}_{j}} p_{j}^{n}+\beta_{i j} p_{j}^{n}:=\alpha_{i j}^{n}, \quad \text { on } \Gamma_{i j}, j \in \mathcal{I}_{i} .
\end{aligned}
$$

3. $n \rightarrow n+1$, go to step 1 .

Here, the parameters $\beta_{i j}$ are freely chosen as positive numbers.

It is obvious that this scheme is consistent in the sense that if we assume convergence then in the limit the transmission conditions (2.33) are satisfied.

\section{Remarks on the well-posedness of the optimality system (2.2), (2.5) and (2.26)}

We now comment on the well-posedness of the optimality system (2.2), (2.5) as well as (2.26). Optimal control problem (2.4) has been investigated by many authors in the past, see e.g. the early work by D. L. Russell [35] in 1960s. Therefore, the well-posedness of the problem along with the optimality systems seems to be mathematical 'folklore' which, in turn, refers to a fixed point argument. However, a concise statement is not easy to localize in the literature. For that matter, we include the arguments briefly. Indeed, problem (2.2) gives rise to a semigroup in the space $\mathcal{H}:=L^{2}(0, L)^{d}$, where we define the operator

$$
\begin{aligned}
\mathcal{L} y & :=\Lambda \partial_{x} y \\
D(\mathcal{L}) & :=\left\{y \in \mathcal{H} \mid \Lambda \partial_{x} y \in \mathcal{H}, \quad\left(\begin{array}{l}
y^{+}(t, 0) \\
y^{-}(t, L)
\end{array}\right)=K\left(\begin{array}{l}
y^{+}(t, L) \\
y^{-}(t, 0)
\end{array}\right)\right\},
\end{aligned}
$$

and its adjoint

$$
\begin{aligned}
\mathcal{L}^{*} p & :=\Lambda \partial_{x} p \\
D\left(\mathcal{L}^{*}\right) & :=\left\{p \in \mathcal{H} \mid \Lambda \partial_{x} p \in \mathcal{H}, \quad\left(\begin{array}{l}
p^{+}(t, L) \\
p^{-}(t, 0)
\end{array}\right)=\tilde{K}\left(\begin{array}{l}
p^{+}(t, 0) \\
p^{-}(t, L)
\end{array}\right)\right\} .
\end{aligned}
$$

The operators $\mathcal{L}$ and $\mathcal{L}^{*}$ are indeed adjoint to each other and it can be seen from [6] [Appendix A] that they generate $C_{0}$-semigroups $\mathcal{S}(t)$ and $\mathcal{S}^{*}(t)$ in $\mathcal{H}$ 
which are then also mutually adjoint to each other and are contractions. Clearly, using the variation of constants formula for semigroups, one can write down the solution $y$ of (2.2) for a given distributed control $u$ as follows

$$
y(t)=\mathcal{S}(t) y_{0}+\int_{0}^{t} \mathcal{S}(t-s) B_{d} u(s) \mathrm{d} s,
$$

while the solution of the adjoint equation (2.5) is given by

$$
p(t)=\int_{T}^{T-t} \mathcal{S}^{*}(s-(T-t)) \kappa\left[y(T-s)-y_{d}(T-s)\right] \mathrm{d} s .
$$

Using (3.3) in (3.4), we arrive at

$$
\begin{aligned}
p(t)= & \kappa \int_{T}^{T-t} \mathcal{S}^{*}(s-(T-t)) \\
& \times\left[\mathcal{S}(T-s) y_{0}+\int_{0}^{T-s} \mathcal{S}(T-s-\tau) B_{d} u(\tau) \mathrm{d} \tau-y_{d}(T-s)\right] \mathrm{d} s \\
= & -\int_{0}^{t} \mathcal{S}^{*}(t-s) \int_{0}^{s} \mathcal{S}(s-\tau) B_{d} u(\tau) \mathrm{d} \tau \mathrm{d} s \\
& -\kappa \int_{0}^{t} \mathcal{S}^{*}(t-s)\left[\mathcal{S}(s) y_{0}-y_{d}(s)\right] \mathrm{d} s .
\end{aligned}
$$

As $u=\frac{1}{\nu} B_{d}^{\top} p(2.7)$, we obtain

$$
\begin{aligned}
u(t)= & -\frac{\kappa}{\nu} \int_{0}^{t} B_{d}^{\top} \mathcal{S}^{*}(t-s) \int_{0}^{s} \mathcal{S}(s-\tau) B_{d} u(\tau) \mathrm{d} \tau \mathrm{d} s \\
& -\kappa \int_{0}^{t} B_{d} \mathcal{S}^{*}(t-s)\left[\mathcal{S}(s) y_{0}-y_{d}(s)\right] \mathrm{d} s
\end{aligned}
$$

and, consequently,

$$
\begin{aligned}
& u+\frac{\kappa}{\nu} \int_{0}^{t} B_{d}^{\top} \mathcal{S}^{*}(t-s) \int_{0}^{s} \mathcal{S}(s-\tau) B_{d} u(\tau) \mathrm{d} \tau \mathrm{d} s \\
& =-\kappa \int_{0}^{t} \mathcal{S}^{*}(t-s)\left[\mathcal{S}(s) y_{0}-y_{d}(s)\right] \mathrm{d} s .
\end{aligned}
$$

Writing the convolution of two functions $w, v$ as $w * v$, denoting

$$
g:=-\kappa \int_{0}^{t} \mathcal{S}^{*}(t-s)\left[\mathcal{S}(s) y_{0}-y_{d}(s)\right] \mathrm{d} s, \quad \mathcal{R}(t):=B_{d}^{\top} \mathcal{S}^{*} * \mathcal{S} B_{d},
$$

and using the associative law for the convolution, we obtain

$$
u+\frac{\kappa}{\nu} \mathcal{R} * u=g .
$$


Equation (3.8) is a Volterra integral equation of second kind which, according to [34] [Thm. 5.1.1], admits a unique solution $u$ which, in turn, shares the regularity of the right hand side. In particular, if we assume $y_{0}, y_{d} \in D(\mathcal{L})$ such that $\partial_{t} y_{d}+\Lambda \partial_{x} y_{d} \in \mathcal{H}$ then $f \in C^{1}$ and, hence, $u \in C^{1}$. We have, thus, the well-known effect of additional regularity for the optimal control. Obviously, we can infer the well-posedness of the optimality system (2.4), (2.5) with $u$ given by (2.7). The regularity of $u$ and, consequently, on $p$ and $y$ make it possible to arrive at weak solutions for (2.26). To be more explicit, we note that the operator $\nabla \cdot(\mathcal{A} \nabla)$ has functions $p \in\left\{p \in L^{2}(\Omega) \mid \partial_{t} p+\Lambda \partial_{x} p=0\right\}=: \mathcal{Z}$ in its kernel. However, the bilinear form

$$
\begin{aligned}
a(p, q) & :=\int_{\Omega}(\nabla q)^{\top} \mathcal{A} \nabla p \mathrm{~d} \omega+\frac{\kappa}{\nu} \int_{\Omega}\left(q^{\top} B_{d}\right)\left(B_{d}^{\top} p\right) \mathrm{d} \omega \\
& =\int_{\Omega}\left(\partial_{t} q+\Lambda \partial_{x} q\right)^{\top}\left(\partial_{t} p+\Lambda \partial_{x} p\right) \mathrm{d} \omega+\frac{\kappa}{\nu} \int_{\Omega}\left(q^{\top} B_{d}\right)\left(B_{d}^{\top} p\right) \mathrm{d} \omega
\end{aligned}
$$

is coercive if ker $B_{d}=\{0\}$, as $a(p, p)$ then is a norm in $\mathcal{H}^{1}=\left\{p \in H^{1}(\Omega)^{2 d}|p|_{\Gamma_{4}}=0\right\}$. In fact, in the sequel, we take $B_{d}=I$. As with Neumann problems, one can always introduce a quotient space $W=H^{1} / \mathcal{Z}$. Operators such as above are sometimes denoted as semi-elliptic, but that notation does not seem to be unique. We close this section with a summary of the observations above.

Proposition 3.1. Let $y_{d}, y_{0} \in D(\mathcal{L})$ such that $\partial_{t} y_{d}+\Lambda \partial_{x} y_{d} \in \mathcal{H}$, then problem (2.26) admits a unique strong solution.

\section{Convergence}

In order to prove convergence of the proposed domain decomposition method described above in the algorithm DDM, we set $B_{d}=I$ and introduce the errors $\tilde{p}_{i}^{n}:=p_{i}^{n}-p_{i}, n \in \mathbb{N}, i \in \mathcal{I}$. As $p^{n}$ and $p$ satisfy the same system with the same boundary conditions at the external boundary, the data for the variables $\tilde{p}_{i}^{n}$ are identical to zero in the state equations as well on the external boundaries. Therefore, $\tilde{p}_{i}^{n}$ satisfies the system (cf. (2.35))

$$
\begin{array}{ll}
-\nabla \cdot\left(\mathcal{A}_{i} \nabla \tilde{p}_{i}^{n+1}\right)+\frac{\kappa}{\nu} \tilde{p}_{i}^{n+1}=0 & \text { in } \Omega, \\
\mathcal{B}_{i 1} \partial_{\nu_{\mathcal{A}_{i}}} \tilde{p}_{i}^{n+1}=0, \quad \mathcal{B}_{i 0} \tilde{p}_{i}^{n+1}=0 & \text { on } \Gamma_{i, e x t}, \\
\partial \nu_{\mathcal{A}_{i}} \tilde{p}_{i}^{n+1}+\beta_{i j} \tilde{p}_{i}^{n}=-\partial \nu_{\mathcal{A}_{j}} \tilde{p}_{j}^{n}+\beta_{i j} \tilde{p}_{j}^{n}:=\alpha_{i j}^{n} & \text { on } \Gamma_{i j}, j \in \mathcal{I}_{i},
\end{array}
$$

where the parameters $\beta_{i j}$ are freely chosen as real positive numbers.

We introduce the space

$$
\mathcal{X}:=\prod_{i=1}^{N} L^{2}\left(\gamma_{i}\right)^{d}
$$


with the norm given by

$$
\|X\|^{2}=\sum_{i \in \mathcal{I}} \sum_{j \in \mathcal{I}_{i}} \frac{1}{2 \beta_{i j}} \int_{\Gamma_{i j}}\left|\alpha_{i j}\right|_{\mathbb{R}^{d}}^{2} \mathrm{~d} \gamma,
$$

where $\mathcal{X} \ni X=\left(\alpha_{i}\right)_{i \in \mathcal{I}}, \quad \alpha_{i}:=\left(\alpha_{i j}\right)_{j \in \mathcal{I}_{i}}$.

We now introduce the operator $\mathcal{T}: \mathcal{X} \rightarrow \mathcal{X}$, such that

$$
\mathcal{T} X=\left\{(\mathcal{T} X)_{i} \mid i \in \mathcal{I}\right\}
$$

where (cf. (4.1))

$$
(\mathcal{T} X)_{i}=\left\{(\mathcal{T} X)_{i j} \mid j \in \mathcal{I}_{i}\right\}, \quad(\mathcal{T} X)_{i j}=\alpha_{i j}=\left.\left(-\partial \nu_{\mathcal{A}_{j}} \tilde{p}_{j}+\beta_{i j} \tilde{p}_{j}\right)\right|_{\Gamma_{i j}} .
$$

Then the iteration (4.1) is equivalent to the fixed point iteration

$$
X^{n+1}=\mathcal{T} X^{n}, \quad n=0,1, \ldots
$$

We compute, omitting the iteration index for a while,

$$
\begin{aligned}
\|X\|_{\mathcal{X}}^{2} & =\sum_{i=1}^{N} \sum_{j \in \mathcal{I}_{i}} \frac{1}{2 \beta_{i j}} \int_{\Gamma_{i j}}\left|\partial \nu_{\mathcal{A}_{i}} p_{i}+\beta_{i j} p_{i}\right|_{\mathbb{R}^{d}}^{2} \mathrm{~d} \gamma \\
& =\sum_{i=1}^{N} \sum_{j \in \mathcal{I}_{i}} \int_{\Gamma_{i j}}\left(\frac{1}{2 \beta_{i j}}\left|\partial \nu_{\mathcal{A}_{i}} p_{i}\right|_{\mathbb{R}^{d}}^{2}+p_{i}^{\top} \partial \nu_{\mathcal{A}_{i}} p_{i}+\frac{\beta_{i j}}{2}\left|p_{i}\right|_{\mathbb{R}^{d}}^{2}\right) \mathrm{d} \gamma \\
\|\mathcal{T} X\|_{\mathcal{X}}^{2} & =\sum_{i=1}^{N} \sum_{j \in \mathcal{I}_{i}} \frac{1}{2 \beta_{i j}} \int_{\Gamma_{i j}}\left|-\partial \nu_{\mathcal{A}_{j}} p_{j}+\beta_{i j} p_{j}\right|_{\mathbb{R}^{d}}^{2} \mathrm{~d} \gamma \\
& =\sum_{i=1}^{N} \sum_{j \in \mathcal{I}_{i}} \int_{\Gamma_{i j}}\left(\frac{1}{2 \beta_{i j}}\left|\partial \nu_{\mathcal{A}_{j}} p_{j}\right|_{\mathbb{R}^{d}}^{2}-p_{j}^{\top} \partial \nu_{\mathcal{A}_{j}} p_{j}+\frac{\beta_{i j}}{2}\left|p_{j}\right|_{\mathbb{R}^{d}}^{2}\right) \mathrm{d} \gamma .
\end{aligned}
$$

This gives

$$
\|\mathcal{T} X\|_{\mathcal{X}}^{2}-\|X\|_{\mathcal{X}}^{2}=-2 \sum_{i=1}^{N} \sum_{j \in \mathcal{I}_{i}} \int_{\Gamma_{i j}} p_{i}^{\top} \partial \nu_{\mathcal{A}_{i}} p_{i} \mathrm{~d} \gamma
$$

We go back to the notation of errors and use the Green identity on the subdomain $\Omega_{i}$

$$
\begin{aligned}
0 & =\int_{\Omega_{i}} \tilde{p}_{i}^{\top}\left(-\nabla \cdot\left(\mathcal{A}_{i} \nabla \tilde{p}_{i}\right)+\frac{\kappa}{\nu} \tilde{p}_{i}\right) \mathrm{d} \omega \\
& =-\int_{\Gamma_{i j}} \tilde{p}_{i}^{\top} \partial_{\nu_{\mathcal{A}_{i}}} \tilde{p}_{i} \mathrm{~d} \gamma+\int_{\Omega_{i}}\left|\partial_{t} \tilde{p}_{i}+\Lambda \partial_{x} \tilde{p}_{i}\right|_{\mathbb{R}^{d}}^{2} \mathrm{~d} \omega+\int_{\Omega_{i}} \frac{\kappa}{\nu} \tilde{p}_{i}^{\top} \tilde{p}_{i} \mathrm{~d} \omega \\
& =-\int_{\Gamma_{i j}} \tilde{p}_{i}^{\top} \partial_{\nu_{\mathcal{A}_{i}}} \tilde{p}_{i} \mathrm{~d} \gamma+a_{i}\left(\tilde{p}_{i}, \tilde{p}_{i}\right) .
\end{aligned}
$$


With (4.9) we obtain in (4.8)

$$
\|\mathcal{T} X\|_{\mathcal{X}}^{2}-\|X\|_{\mathcal{X}}^{2}=-2\left\{\sum_{i=1}^{N} \int_{\Omega_{i}}\left|\partial_{t} \tilde{p}_{i}+\Lambda \partial_{x} \tilde{p}_{i}\right|_{\mathbb{R}^{d}}^{2} \mathrm{~d} \omega+\int_{\Omega_{i}} \frac{\kappa}{\nu} \tilde{p}_{i}^{\top} \tilde{p}_{i} \mathrm{~d} \omega\right\},
$$

or, in other words,

$$
\|\mathcal{T} X\|_{\mathcal{X}}^{2}-\|X\|_{\mathcal{X}}^{2}=-2 \sum_{i=1}^{N} a_{i}\left(\tilde{p}_{i}, \tilde{p}_{i}\right) .
$$

Relation (4.11) clearly shows that $\mathcal{T}$ is dissipative. Now instead of the basic iteration (4.5), we introduce the following under-relaxation of (4.5).

$$
X^{n+1}=(1-\epsilon) \mathcal{T} X^{n}+\epsilon X^{n}, \quad \epsilon \in[0,1) .
$$

This together with (4.11) gives

$$
\begin{aligned}
\left\|X^{n+1}\right\|_{\mathcal{X}}^{2} & =\left[(1-\epsilon)^{2}+\epsilon^{2}\right]\left\|X^{n}\right\|_{\mathcal{X}}^{2}+2 \epsilon(1-\epsilon)\left(X^{n}, \mathcal{T} X^{n}\right)_{\mathcal{X}} \\
& -2(1-\epsilon)^{2} \sum_{i=1}^{N} a_{i}\left(\tilde{p}_{i}, \tilde{p}_{i}\right) .
\end{aligned}
$$

With the definition

$$
E^{n}:=\sum_{i=1}^{N} \sum_{j \in \mathcal{I}_{i}} \frac{1}{2 \beta_{i j}} \int_{\Gamma_{i j}}\left(\left|\partial \nu_{\mathcal{A}_{i}} \tilde{p}_{i}^{n}\right|_{\mathbb{R}^{d}}^{2}+\beta_{i j}^{2}\left|\tilde{p}_{i}^{n}\right|_{\mathbb{R}^{d}}^{2}\right) \mathrm{d} \gamma
$$

we obtain from this and (4.6)

$$
\left\|X^{n}\right\|_{\mathcal{X}}^{2}=E^{n}+\sum_{i=1}^{N} a_{i}\left(\tilde{p}_{i}, \tilde{p}_{i}\right)=: E^{n}+F^{n}, \quad\left\|\mathcal{T} X^{n}\right\|^{2}=E^{n}-F^{n} .
$$

A straightforward calculation shows

$$
\left(X^{n}, \mathcal{T} X^{n}\right) \leqslant E^{n}
$$

and moreover

$$
E^{n+1} \leqslant E^{n}-\sum_{i=1}^{N}\left[(1-2 \epsilon) a_{i}\left(\tilde{p}_{i}^{n}, \tilde{p}_{i}^{n}\right)+a_{i}\left(\tilde{p}_{i}^{n+1}, \tilde{p}_{i}^{n+1}\right)\right] .
$$

With the coefficients $c_{1}(\epsilon):=1-2 \epsilon, c_{n+1}(\epsilon)=1, c_{l}(\epsilon)=2(1-\epsilon), l=2, \ldots, n$, we obtain from (4.16) the crucial inequality

$$
E^{n+1}+\sum_{l=1}^{n+1} c_{l}(\epsilon) \sum_{i=1}^{l} a_{i}\left(\tilde{p}_{i}^{n}, \tilde{p}_{i}^{n}\right) \leqslant E^{1} .
$$


This inequality implies

$$
\sum_{i=1}^{N} a_{i}\left(\tilde{p}_{i}^{n}, \tilde{p}_{i}^{n}\right) \rightarrow 0
$$

and $E^{n} \leqslant C$ for some $C>0$. If we recall the form $a_{i}(\cdot, \cdot)$ then (4.18) reads as

$$
\sum_{i=1}^{N}\left\{\int_{\Omega_{i}}\left|\partial_{t} \tilde{p}_{i}+\Lambda \partial_{x} \tilde{p}_{i}\right|_{\mathbb{R}^{d}}^{2} \mathrm{~d} \omega+\int_{\Omega_{i}} \frac{\kappa}{\nu} \tilde{p}_{i}^{\top} \tilde{p}_{i} \mathrm{~d} \omega\right\} \rightarrow 0 .
$$

However, the form $a_{i}(\cdot, \cdot)$ is not elliptic but rather positive semi-definite. Therefore, from (4.19), we may conclude

$$
\tilde{p}_{i}^{n} \rightarrow 0, \quad \text { strongly in } L^{2}\left(\Omega_{i}\right)^{d},
$$

while

$$
\partial_{t} \tilde{p}_{i}+\Lambda \partial_{x} \tilde{p}_{i} \rightarrow 0, \quad \text { strongly in } L^{2}\left(\Omega_{i}\right)^{d},
$$

together with (4.20) does, however, not imply $\tilde{p}_{i}^{n} \rightarrow 0$, strongly in $H^{1}\left(\Omega_{i}\right)$. Now, as $E^{n}$ is bounded, we can extract sub-sequences from $\partial \nu_{\mathcal{A}_{i}} \tilde{p}_{i}^{n}, \tilde{p}_{i}^{n}$ on $\Gamma_{i j}$ such that $\left.\partial \nu_{\mathcal{A}_{i}} \tilde{p}_{i}^{n}\right|_{\Gamma_{i j}} \rightarrow q,\left.\tilde{p}_{i}^{n}\right|_{\Gamma_{i j}} \rightarrow r$, weakly in $L^{2}\left(\gamma_{i}\right)^{d}$. Then we may use the Green identity with a test function $\phi$ on $\Omega_{i}$ to conclude that (4.20), (4.21) imply $q=r=0$. But this holds on subsequences which may not contain to consecutive indices $n, n+1$ as required in the iteration. We may take now advantage of the underrelaxation parameter $\epsilon \in(0,1)$.

Proposition 4.1 (Opial [33]). Let $\mathcal{T}$ be non-expansive with at least one fixed point. Then for each $\epsilon \in(0,1)$ the sequence $\left\{\mathcal{T}_{\epsilon}^{n} X\right\}$ is weakly convergent to a fixed point.

Here we have set $\mathcal{T}_{\epsilon}:=\epsilon I+(1-\epsilon) \mathcal{T}$. As we have seen, our map $\mathcal{T}$ is nonexpansive and 0 is in fact the unique fixed point. Thus, we may conclude that entire sequences converge to zero and, moreover, $X^{n} \rightarrow 0$ and $\mathcal{T} X^{n} \rightarrow 0$. Now, in order to prove strong convergence of $\left\|\mathcal{T} X^{n}-X^{n}\right\|_{\mathcal{X}} \rightarrow 0$, we are going to apply Schaefer's theorem [5]. To this end, we recall the definition of an asymptotic regular map $\mathcal{T}$. Let $\mathcal{C} \subset \mathcal{X}$ closed and convex and $\mathcal{T}$ non-expansive. Then $\mathcal{T}$ is said to be asymptotically regular if for any $X \in \mathcal{C}$ the sequence $\left\{\mathcal{T}^{n+1} X-\mathcal{T}^{n} X\right\}$ tends to zero as $n \rightarrow \infty$.

Proposition 4.2 (Schaefer [5]). If $\mathcal{T}$ has at least one fixed point in $\mathcal{C}$, then the mapping $\mathcal{T}_{\epsilon}$ is asymptotically regular.

From this and (4.15), we infer

$$
\left\|\mathcal{T} X^{n}-X^{n}\right\|_{\mathcal{X}}^{2}=2 E^{n}-2\left(X^{n}, \mathcal{T} X^{n}\right) \rightarrow 0 .
$$


We calculate the second term

$$
\begin{aligned}
&\left(X^{n}, \mathcal{T} X^{n}\right)= \sum_{i=1}^{N} \sum_{j \in \mathcal{I}_{i}} \frac{1}{2 \beta_{i j}} \int_{\Gamma_{i j}}\left(-\partial \nu_{\mathcal{A}_{j}} \tilde{p}_{j}^{n}+\beta_{i j} \tilde{p}_{j}^{n}\right)^{\top}\left(\partial \nu_{\mathcal{A}_{i}} \tilde{p}_{i}^{n}+\beta_{i j} \tilde{p}_{i}^{n}\right) \mathrm{d} \gamma \\
&=\sum_{i=1}^{N} \sum_{j \in \mathcal{I}_{i}} \frac{1}{2 \beta_{i j}} \int_{\Gamma_{i j}}\left\{-\left(\partial \nu_{\mathcal{A}_{j}} \tilde{p}_{j}^{n}\right)^{\top}\left(\partial \nu_{\mathcal{A}_{i}} \tilde{p}_{i}^{n}\right)+\beta_{i j}^{2}\left(\tilde{p}_{j}^{n}\right)^{\top}\left(\tilde{p}_{i}^{n}\right)\right. \\
&\left.\quad+\beta_{i j}\left[\left(\tilde{p}_{j}^{n}\right)^{\top}\left(\partial \nu_{\mathcal{A}_{i}} \tilde{p}_{i}^{n}\right)-\left(\partial \nu_{\mathcal{A}_{j}} \tilde{p}_{j}^{n}\right)^{\top}\left(\tilde{p}_{i}^{n}\right)\right]\right\} \mathrm{d} \gamma \\
&=\sum_{i=1}^{N} \sum_{j \in \mathcal{I}_{i}} \frac{1}{2 \beta_{i j}} \int_{\Gamma_{i j}}\left[\beta_{i j}^{2}\left(\tilde{p}_{j}^{n}\right)^{\top}\left(\tilde{p}_{i}^{n}\right)-\left(\partial \nu_{\mathcal{A}_{j}} \tilde{p}_{j}^{n}\right)^{\top}\left(\partial \nu_{\mathcal{A}_{i}} \tilde{p}_{i}^{n}\right)\right] \mathrm{d} \gamma
\end{aligned}
$$

We obtain from this

$$
2 E^{n}-2\left(X^{n}, \mathcal{T} X^{n}\right)=\sum_{i=1}^{N-1} \sum_{\substack{j \in \mathcal{I}_{i} \\ j>i}} \int_{\Gamma_{i j}}\left(\frac{1}{\beta_{i j}}\left|\partial \nu_{\mathcal{A}_{i}} \tilde{p}_{i}^{n}+\partial \nu_{\mathcal{A}_{j}} \tilde{p}_{j}^{n}\right|^{2}+\beta_{i j}\left|\tilde{p}_{i}^{n}-\tilde{p}_{j}^{n}\right|^{2}\right) \mathrm{d} \gamma
$$

In conclusion, from (4.22) and (4.24), we obtain for all $i, j: j \in \mathcal{I}_{i}$

$$
\begin{aligned}
&\left|\partial \nu_{\mathcal{A}_{i}} \tilde{p}_{i}^{n}+\partial \nu_{\mathcal{A}_{j}} \tilde{p}_{j}^{n}\right|_{L^{2}\left(\Gamma_{i j}\right)} \rightarrow 0, n \rightarrow \infty, \\
&\left|\tilde{p}_{i}^{n}-\tilde{p}_{j}^{n}\right|_{L^{2}\left(\Gamma_{i j}\right)} \rightarrow 0, \quad n \rightarrow \infty .
\end{aligned}
$$

We have therefore proved our main

Theorem 4.1. Let $\epsilon \in[0,1)$ be given. Then the iteration defined in (4.1) converges in the following sense:

$$
\begin{aligned}
& \text { 1) } \epsilon=0
\end{aligned}
$$

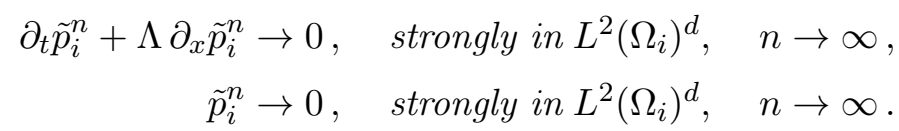

On a subsequence we have

$$
\begin{aligned}
& \partial \nu_{\mathcal{A}_{i}} \tilde{p}_{i}^{n} \rightarrow 0, \quad \text { weakly in } L^{2}\left(\Gamma_{i j}\right)^{d}, \quad n \rightarrow \infty, \\
& \tilde{p}_{i}^{n} \rightarrow 0, \quad \text { weakly in } L^{2}\left(\Gamma_{i j}\right)^{d}, \quad n \rightarrow \infty .
\end{aligned}
$$

2) $\epsilon \in(0,1)$ In addition to case 1$)$, we have

$$
\begin{aligned}
\partial \nu_{\mathcal{A}_{i}} \tilde{p}_{i}^{n}+\partial \nu_{\mathcal{A}_{j}} \tilde{p}_{j}^{n} \rightarrow 0, & \text { strongly in } L^{2}\left(\Gamma_{i j}\right)^{d}, & & n \rightarrow \infty, \\
\tilde{p}_{i}^{n}-\tilde{p}_{j}^{n} \rightarrow 0, & \text { strongly in } L^{2}\left(\Gamma_{i j}\right)^{d}, & & n \rightarrow \infty .
\end{aligned}
$$

Thus, the iterates $p_{i}^{n}$ converge to the restriction of solution $\left.p\right|_{\Omega_{i}}$ in Algorithm $D D M(2.35)$ in the sense of 1) and 2), respectively. 
Remark 4.1. We can interpret Algorithm DDM (2.35) in the framework of the original problem (2.2). Clearly, (4.26) for $\epsilon=0$ implies

$$
\begin{aligned}
\tilde{y}_{i}^{n}, \tilde{p}_{i}^{n} \rightarrow 0, & \text { strongly in } L^{2}\left(\Omega_{i}\right)^{d}, & & n \rightarrow \infty, \\
\left.\tilde{y}_{i}^{n}\right|_{L^{2}\left(\Gamma_{i j}\right)},\left.\tilde{p}_{i}^{n}\right|_{L^{2}\left(\Gamma_{i j}\right)} \rightarrow 0, & \text { weakly in } L^{2}\left(\Omega_{i}\right)^{d}, & & n \rightarrow \infty .
\end{aligned}
$$

Moreover, in case $\epsilon \in(0,1)$, we can, in addition to (4.28), infer from (4.27)

$$
\left.\left(\tilde{y}_{i}^{n}-\tilde{y}_{j}^{n}\right)\right|_{L^{2}\left(\Gamma_{i j}\right)},\left.\left(\tilde{p}_{i}^{n}-\tilde{p}_{j}^{n}\right)\right|_{L^{2}\left(\Gamma_{i j}\right)} \rightarrow 0, \quad \text { strongly in } L^{2}\left(\Gamma_{i j}\right)^{d}, \quad n \rightarrow \infty .
$$

\section{A posteriori error estimates}

We now embark on a posteriori error estimates. For the sake of simplicity, we confine ourselves with two subdomains, either horizontal or vertical. So both cases, i.e. time- and space decomposition are covered in principle. We follow the ideas of [29] for scalar elliptic equations and emphasize that we have to deal with systems of semi-elliptic equations. Therefore the arguments deserve a special treatment. We now take two subdomains $\Omega_{i}, i=1,2$ but still use the notation above. We recall the quadratic forms

$$
\begin{gathered}
a(p, q)=a_{\Omega}(p, v):=\int_{\Omega}\left[(\nabla v)^{\top}(\mathcal{A} \nabla p)+\frac{\kappa}{\nu} v^{\top} p\right] \mathrm{d} \omega \\
a_{i}\left(p_{i}, q_{i}\right)=a_{\Omega_{i}}\left(p_{i}, v_{i}\right):=\int_{\Omega_{i}}\left[\left(\nabla v_{i}\right)^{\top}\left(\mathcal{A}_{i} \nabla p_{i}\right)+\frac{\kappa}{\nu} v_{i}^{\top} p_{i}\right] \mathrm{d} \omega .
\end{gathered}
$$

Under the conditions on the target function $y_{d}$, we can rewrite the boundary value problem in terms of the above quadratic forms

$$
a(p, v)=\kappa(f, v), \quad \sum_{i=1}^{2} a_{i}\left(p_{i}, v_{i}\right)=\sum_{i=1}^{2} \kappa\left(f_{i}, v_{i}\right) .
$$

We introduce $\Gamma=\Gamma_{12}=\Gamma_{21}, \beta_{i j}=\beta_{j i}=\beta_{i}$ and the space $V:=\left\{p \in H^{1}(\Omega)^{d} \mid\right.$ $B_{i 0} p=0$ on $\left.\Gamma_{i}, i=1,2,4\right\}, V_{i}=H^{1}\left(\Omega_{i}\right)^{d} \cap V, W:=\left\{\left.p\right|_{\Gamma} \mid p \in V\right\} \subset L^{2}(\Gamma)^{d}$. We have a bounded trace operator and its pre-images

$$
\operatorname{tr}_{i}: V_{i} \rightarrow L^{2}(\Gamma)^{d}, \quad \operatorname{tr}_{i}^{-1}: W \rightarrow V_{i}
$$

such that $v_{i} \in V_{i}$ can be expressed as $v_{i}=\operatorname{tr}_{i}^{-1} \phi+v_{0 i}, \phi \in W, v_{0 i} \in V_{i} \cap H_{0}^{1}\left(\Omega_{i}\right)^{d}$. Hence,

$$
a_{i}\left(p_{i}, v_{i}\right)+\int_{\Gamma} \beta_{i} v_{i}^{\top} p_{i} \mathrm{~d} \gamma=\int_{\Gamma} v_{i}^{\top} \alpha_{i} \mathrm{~d} \gamma+\kappa \int_{\Omega_{i}} v_{i}^{\top} f_{i} \mathrm{~d} \omega .
$$

According to the boundary conditions at the interface $\Gamma$, we obtain

$$
\alpha_{i}^{n+1}+\alpha_{j}^{n}=\left(\beta_{i}+\beta_{j}\right) p_{j}^{n+1} .
$$


This allows us to rewrite the local boundary value problem as

$$
\begin{array}{ll}
a_{i}\left(p_{i}^{n+1}, v_{i}\right)+\left.\beta_{i}\left(p_{i}^{n+1}, v_{i}\right)\right|_{\Gamma}=\left.\left(\alpha_{i}^{n}, v_{i}\right)\right|_{\Gamma}+\kappa\left(f_{i}, v_{i}\right), & v_{i} \in V_{i}, \\
\left.\left(\alpha_{i}^{n+1}, \phi\right)\right|_{\Gamma}=\left.\left(\beta_{i}+\beta_{j}\right)\left(p_{j}^{n+1}, \phi\right)\right|_{\Gamma}-\left.\left(\alpha_{j}^{n}, \phi\right)\right|_{\Gamma}, & \phi \in W .
\end{array}
$$

When we take the errors $\tilde{p}_{i}^{n}:=p_{i}^{n}-p_{i}$ and $\tilde{\alpha}_{i}^{n}:=\alpha_{i}^{n}-\alpha_{i}$, then (5.4) turns into

$$
\begin{aligned}
& a_{i}\left(\tilde{p}_{i}^{n+1}, \operatorname{tr}_{i}^{-1} \phi\right)+\left.\beta_{i}\left(\tilde{p}_{i}^{n+1}, \operatorname{tr}_{i}^{-1} \phi\right)\right|_{\Gamma}=\left.\left(\tilde{\alpha}_{i}^{n}, \operatorname{tr}_{i}^{-1} \phi\right)\right|_{\Gamma}, \quad \phi \in W, \\
& \left.\left(\tilde{\alpha}_{i}^{n+1}, \phi\right)\right|_{\Gamma}=\left.\left(\beta_{i}+\beta_{j}\right)\left(\tilde{p}_{j}^{n+1}, \phi\right)\right|_{\Gamma}-\left.\left(\tilde{\alpha}_{j}^{n}, \phi\right)\right|_{\Gamma},
\end{aligned}
$$

and we obtain

$$
\left.\left(\tilde{\alpha}_{i}^{n-1}-\beta_{i} \tilde{p}_{i}^{n}, \phi\right)\right|_{\Gamma}=a_{i}\left(\tilde{p}_{i}^{n}, \operatorname{tr}_{i}^{-1} \phi\right) \leqslant C\left\|\tilde{p}_{i}^{n}\right\|\|\phi\|,
$$

where $C>0$ is a computable number depending on the norm of $\operatorname{tr}_{i}^{-1}, \mathcal{A}_{i}$ and $\kappa, \nu$. Similarly,

$$
\left.\left(\beta_{i} \phi, \tilde{p}_{i}^{n+1}\right)\right|_{\Gamma} \leqslant C_{W}\left\|\tilde{p}_{i}^{n+1}\right\|\|\phi\|
$$

We compute

$$
\begin{aligned}
a_{1}\left(\tilde{p}_{1}^{n+1}, v_{1}\right) & +a_{2}\left(\tilde{p}_{2}^{n}, v_{2}\right)=\left.\left(\tilde{\alpha}_{2}^{n}-\beta_{1} \tilde{p}_{1}^{n+1}, v_{1}\right)\right|_{\Gamma}+\left.\left(\tilde{\alpha}_{1}^{n-1}-\beta_{2} \tilde{p}_{2}^{n}, v_{2}\right)\right|_{\Gamma} \\
& =\left.\left(\beta_{1}\left(\tilde{p}_{2}^{n}-\tilde{p}_{1}^{n+1}\right), v_{1}\right)\right|_{\Gamma}+\left.\left(\tilde{\alpha}_{2}^{n}-\beta_{1} \tilde{p}_{2}^{n}, v_{1}\right)\right|_{\Gamma} \\
& +\left.\left(\tilde{\alpha}_{1}^{n-1}-\beta_{2} \tilde{p}_{2}^{n}, v_{2}-v_{1}\right)\right|_{\Gamma}+\left.\left(\tilde{\alpha}_{1}^{n-1}-\beta_{2} \tilde{p}_{2}^{n}, v_{1}\right)\right|_{\Gamma} \\
& =\left.\left(\beta_{1}\left(p_{2}^{n}-p_{1}^{n+1}\right), v_{1}\right)\right|_{\Gamma}+\left.\left(\tilde{\alpha}_{1}^{n-1}-\beta_{2} \tilde{p}_{2}^{n}, v_{2}-v_{1}\right)\right|_{\Gamma}
\end{aligned}
$$

We now take as test functions $v_{1}=\tilde{p}_{1}^{n+1}, v_{2}=\tilde{p}_{2}^{n}$ in (5.8) in order to obtain

$$
\begin{aligned}
a_{1}\left(\tilde{p}_{1}^{n+1}, \tilde{p}_{1}^{n+1}\right) & +a_{2}\left(\tilde{p}_{2}^{n}, \tilde{p}_{2}^{n}\right) \\
& =\left.\left(\beta_{1}\left(p_{2}^{n}-p_{1}^{n+1}\right), \tilde{p}_{1}^{n+1}\right)\right|_{\Gamma}+\left.\left(\tilde{\alpha}_{1}^{n-1}-\beta_{2} \tilde{p}_{2}^{n}, \tilde{p}_{2}^{n}-\tilde{p}_{1}^{n+1}\right)\right|_{\Gamma} \\
& \leqslant C\left(\left\|\tilde{p}_{1}^{n+1}\right\|_{L^{2}(\Gamma)^{d}}+\left\|\tilde{p}_{2}^{n}\right\|_{L^{2}(\Gamma)^{d}}\right)\left\|p_{2}^{n}-p_{1}^{n+1}\right\|_{L^{2}(\Gamma)^{d}}
\end{aligned}
$$

where again $C$ is a computable number involving the previous constants and again the norms of tr, $\mathcal{A}$. Notice that due to our convergence result, if $\epsilon \in(0,1)$, the trace $\tilde{p}_{i}^{n}$ strongly converge to zero and the sequences are, hence, bounded. We do not have ellipticity of the forms in general, but due to the penalty term $\frac{\kappa}{\nu}>0$ we have the following a posteriori error estimate.

Theorem 5.1. Let the iterates $p_{i}^{n}, i=1,2$ be the solutions of $(2.35)$ for $\epsilon \in(0,1)$ and let $p_{i}$ solve the original optimality system (2.26), then

$$
\left\|p_{1}^{n+1}-p_{1}\right\|_{L^{2}\left(\Omega_{1}\right)^{d}}+\left\|p_{2}^{n}-p_{2}\right\|_{L^{2}\left(\Omega_{2}\right)^{d}} \leqslant C\left|p_{1}^{n+1}-p_{2}^{n}\right|_{L^{2}(\Gamma)^{d}}^{\frac{1}{2}} .
$$

Remark 5.1. We notice that the error estimate (5.10) is weaker than the corresponding one for elliptic problems $[3,29]$. 


\section{Interpretation for the original system}

If we restrict ourselves to domain decomposition just w.r.t the time variable, we obtain from transmission condition in Algorithm DDM (2.35), with $\kappa \beta_{14}$ $=\kappa \beta_{41}=\beta$ precisely the time-domain decomposition discussed in [32]. If, however we concentrate on just spatial domain decomposition, we need to rewrite the iteration for $y_{i}$ and $p_{i}$ in terms of $y_{i}^{+}, y_{i}^{-}$and $p_{i}^{+}, p_{i}^{-}$in order to have well-posed subproblems. We have first from transmission condition for $p$

$$
\begin{aligned}
-\Lambda\left(\partial_{t} p_{2}+\Lambda \partial_{x} p_{2}\right)+\beta_{12} p_{2} & =-\Lambda\left(\partial_{t} p_{1}+\Lambda \partial_{x} p_{1}\right)+\beta_{12} p_{1}, \\
\Lambda\left(\partial_{t} p_{1}+\Lambda \partial_{x} p_{1}\right)+\beta_{21} p_{1} & =\Lambda\left(\partial_{t} p_{2}+\Lambda \partial_{x} p_{2}\right)+\beta_{21} p_{2},
\end{aligned}
$$

which translates at the breakpoint $x=x_{1}$ (of the continuous matching) to

$$
\begin{aligned}
-\kappa \Lambda^{+} y_{2}^{+}+\beta_{12} p_{2}^{+} & =-\kappa \Lambda^{+} y_{1}^{+}+\beta_{12} p_{1}^{+}, \\
-\kappa \Lambda^{-} y_{2}^{-}+\beta_{12} p_{2}^{-} & =-\kappa \Lambda^{-} y_{1}^{-}+\beta_{12} p_{1}^{-}, \\
\kappa \Lambda^{+} y_{1}^{+}+\beta_{21} p_{1}^{+} & =\kappa \Lambda^{+} y_{2}^{+}+\beta_{21} p_{2}^{+}, \\
\kappa \Lambda^{-} y_{1}^{-}+\beta_{21} p_{1}^{-} & =\kappa \Lambda^{-} y_{2}^{-}+\beta_{21} p_{2}^{-} .
\end{aligned}
$$

We can describe $y_{2}^{+}\left(x_{1}\right), p_{2}^{-}\left(x_{1}\right)$ and $y_{1}^{-}\left(x_{1}\right), p_{1}^{+}\left(x_{1}\right)$. Thus, we write

$$
\begin{aligned}
\left(\begin{array}{r}
-\kappa \Lambda^{+} y_{2}^{+} \\
\beta_{21} p_{2}^{-}
\end{array}\right) & =-\left(\begin{array}{r}
\beta_{12} p_{2}^{+} \\
-\kappa \Lambda^{-} y_{2}^{-}
\end{array}\right)+\left(\begin{array}{l}
\alpha_{12}^{+} \\
\alpha_{12}^{-}
\end{array}\right), \\
\left(\begin{array}{r}
\beta_{21} p_{1}^{+} \\
+\kappa \Lambda^{-} y_{1}^{-}
\end{array}\right) & =-\left(\begin{array}{r}
\beta_{12} p_{2}^{+} \\
-\kappa \Lambda^{-} y_{2}^{-}
\end{array}\right)+\left(\begin{array}{l}
\alpha_{12}^{+} \\
\alpha_{12}^{-}
\end{array}\right) .
\end{aligned}
$$

If we proceed from the original problem (2.2) and the adjoint problem (2.5) and use the corresponding Green identity on the space-time domain, we obtain, by the way of variations, transmission conditions for the state $y$ and the adjoint variable $p$ at the artificial interfaces $\Gamma_{i j}$. In the special case of spatial domain decomposition discussed above, we have two domains $\Omega_{1}:=\left\{(t, x) \mid t \in(0, T) \times\left(0, x_{1}\right)\right\}$, $\Omega_{2}:=\left\{(t, x) \mid t \in(0, T) \times\left(x_{1}, L\right)\right\}$, then at $\Gamma_{12}:=\left\{\left(t, x_{1}\right) \mid t \in(0, T)\right\}$ we have

$$
\begin{array}{ll}
y_{2}^{+}\left(t, x_{1}\right)=y_{1}^{+}\left(t, x_{1}\right), & y_{2}^{+}\left(t, x_{1}\right)=y_{1}^{+}\left(t, x_{1}\right), \\
p_{2}^{+}\left(t, x_{1}\right)=p_{1}^{+}\left(t, x_{1}\right), & p_{1}^{+}\left(t, x_{1}\right)=p_{2}^{+}\left(t, x_{1}\right) .
\end{array}
$$

These conditions are also clear from the point of view of the characteristics: for example, the outgoing information $y_{1}^{+}\left(t, x_{1}\right)$ at the right boundary of the first domain matches the incoming information $y_{2}^{+}\left(t, x_{1}\right)$ at the left boundary of the second domain. In order to address a domain decomposition at this level directly, we would stipulate the ansatz (6.2) also directly coming from the original problem formulation in terms of $y$ and $p$. The reformulated boundary expressions (6.3) show 
that on the level of the decomposed optimality system the boundary conditions lead to a well posed system on the individual subdomain $\Omega_{i}$. We then we introduce the corresponding iteration

$$
\begin{aligned}
-\kappa \Lambda^{+}\left(y_{2}^{+}\right)^{n+1}+\beta_{12}\left(p_{2}^{+}\right)^{n+1} & =-\kappa \Lambda^{+}\left(y_{1}^{+}\right)^{n}+\beta_{12}\left(p_{1}^{+}\right)^{n}=\alpha_{21}^{+, n}, \\
-\kappa \Lambda^{-}\left(y_{2}^{-}\right)^{n+1}+\beta_{12}\left(p_{2}^{-}\right)^{n+1} & =-\kappa \Lambda^{-}\left(y_{1}^{-}\right)^{n}+\beta_{12}\left(p_{1}^{-}\right)^{n}=\alpha_{21}^{-, n}, \\
\kappa \Lambda^{+}\left(y_{1}^{+}\right)^{n+1}+\beta_{21}\left(p_{1}^{+}\right)^{n+1} & =\kappa \Lambda^{+}\left(y_{2}^{+}\right)^{n}+\beta_{21}\left(p_{2}^{+}\right)^{n}=\alpha_{12}^{+, n}, \\
\kappa \Lambda^{-}\left(y_{1}^{-}\right)^{n+1}+\beta_{21}\left(p_{1}^{-}\right)^{n+1} & =\kappa \Lambda^{-}\left(y_{2}^{-}\right)^{n}+\beta_{21}\left(p_{2}^{-}\right)^{n}=\alpha_{12}^{-, n} .
\end{aligned}
$$

In order to understand the time-space decomposition of the original problem, we focus on a subdomain $\Omega_{i}$ with four neighbours $\Omega_{j}, j \in \mathcal{I}_{i}$. We need to distinguish the horizontal neighbours $j \in \mathcal{I}_{i}^{x}$ leading to a spatial decomposition and vertical neighbours $j \in \mathcal{I}_{i}^{t}$ representing the time-domain decomposition. Accordingly, we introduce $S_{i j}=I, j \in \mathcal{I}_{i}^{t}$ and $S_{i j}=\kappa \Lambda, j \in \mathcal{I}_{i}^{x}$. Moreover, we introduce the signs $\epsilon_{i j}=1, j \in \mathcal{I}_{i}^{x}$ if $j$ signifies the domain $\Omega_{j}$ right to $\Omega$, whereas $\epsilon_{i j}=-1$ if $\Omega_{j}$ is left to it. Similarly, for the upper and lower neighbours of $\Omega_{i}$. With this notation, the transmission conditions of (2.35), written there in terms of $p$ can now be reformulated as

$$
\epsilon_{i j} S_{i j} y_{i}^{n+1}+\beta_{i j} p_{i}^{n+1}=-\epsilon_{j i} S_{j i} y_{j}^{n}+\beta_{i j} p_{i}^{n}=: \alpha_{i j}^{n}, \quad \text { on } \Gamma_{i j}, \forall j \in \mathcal{I}_{i} .
$$

The question that we want to address now is whether this decomposition of the optimality system in terms of $y$ and $p$ can be interpreted as the optimality system associated with an optimal control problem on the subdomains. With the formulation (6.6) this task is now rather straightforward. Indeed, let us introduce virtual controls $h_{i j} \in L^{2}\left(\Gamma_{i j}\right)^{d}, j \in \mathcal{I}_{i}$. We introduce the optimal control problem

$$
\left\{\begin{aligned}
\min J\left(u_{i}, y_{i}, h_{i j}\right):= & \frac{\kappa}{2} \int_{\Omega_{i}}\left\|y_{i}-y_{d, i}\right\|^{2} \mathrm{~d} \omega+\frac{\nu}{2} \int_{\Omega_{i}} \| u_{i}^{2} \mathrm{~d} \omega \\
+ & \sum_{j \in \mathcal{I}_{i}} \int_{\Gamma_{i j}} \frac{1}{2 \beta_{i j}}\left(\left|h_{i j}\right|^{2}+\left|\alpha_{i j}\right|^{2}\right) \mathrm{d} \gamma, \\
\text { s.t. } & \begin{cases}\partial_{t} y_{i}+\Lambda \partial_{x} y_{i}=u_{i}, & \text { in } \Omega_{i}, \\
\epsilon_{i j} S_{i j} y_{i}=\alpha_{i j}+h_{i j}, & \text { on } \Gamma_{i j}, j \in \mathcal{I}_{i} .\end{cases}
\end{aligned}\right.
$$

Remark 6.1 . We note that the boundary value problem in (6.7) is overdetermined and thus formally ill-posed. However, the virtual control $h_{i j}$ turns the optimal control problem (6.7) into a well-posed optimization problem on each subdomain $\Omega_{i}$.

If we put the data $\alpha_{i j}$ and the distributed as well as the virtual control at iteration index $n+1$, then the corresponding (well-posed) optimality system is

$$
\begin{aligned}
\partial_{t} y_{i}^{n+1}+\Lambda \partial_{x} y_{i}^{n+1} & =\frac{1}{\nu} p_{i}^{n+1}, & & \text { in } \Omega_{i}, \\
\partial_{t} p_{i}^{n+1}+\Lambda \partial_{x} p_{i}^{n+1} & =\kappa\left(y_{i}^{n+1}-y_{i d}\right), & & \text { in } \Omega_{i}, \\
\epsilon_{i j} S_{i j} y_{i}^{n+1}+\beta_{i j} p_{i}^{n+1} & =-\epsilon_{j i} S_{j i} y_{j}^{n}+\beta_{i j} p_{i}^{n}=\alpha_{i j}^{n}, & & \text { on } \Gamma_{i j}, \forall j \in \mathcal{I}_{i},
\end{aligned}
$$


which can now be reformulated in (also the well-posed) format (2.35). We may, thus, conclude that our decomposed optimality system is itself an optimality system for local virtual control problem on the subdomain $\Omega_{i}, i \in \mathcal{I}$. This is a crucial property, as it implies that we can actually decompose the original optimal control problem to optimal control problems on the subdomains $\Omega_{i}$ !

\section{Conclusions}

We have shown that the classical iterative domain decomposition due to P. L. Lions [25] designed for elliptic scalar equations can be extended to a system of semi-ellptic equations. We include the proof of convergence and provide a variant of a classical a posteriori error estimate [29] for such indefinite elliptic systems. Further, we provide an interpretation in terms of the original optimal control problem for the first order linear hyperbolic systems with constant coefficients. In that context, the algorithm appears as a space-time-domain decomposition method with simultaneous convergence properties. The question of simultaneous convergence has been raised in [2] and can, for the special system considered here, answered affirmatively. More general control input operators are at order and boundary controls should be addressed, too. Space-time-domain decomposition of semi-linear systems such as in $[31,32]$ will be the subject of a forthcoming publication. Also the numerical realization is in preparation.

\section{Acknowledgments}

The author thanks the Deutsche Forschungsgemeinschaft for their support within projects A05 of the Sonderforschungsbereich / Transregio 154 Mathematical Modelling, Simulation and Optimization using the Example of Gas Networks.

\section{References}

1. M. Brokate, Necessary optimality conditions for the control of semilinear hyperbolic boundary value problems, SIAM Journal on Control and Optimization, 25(5) (1985), 1353-1369.

2. J. E. Lagnese, G. LeUgering, Time-domain decomposition of optimal control problems for the wave equation, Systems \& Control Letters, 48(3-4) (2003), 229-242.

3. J. E. Lagnese, G. Leugering, Domain decomposition methods in optimal control of partial differential equations, International Series of Numerical Mathematics, Birkhäuser-Verlag, 148, 2004.

4. Lagnese, John E And Leugering, GÜNTER, Time domain decomposition in final value optimal control of the Maxwell system, ESAIM: Control, Optimisation and Calculus of Variations 8 (2002), 775-799.

5. H. Schaefer, Über die Methode sukzessiver Approximationen, Jahresbericht der Deutschen Mathematiker-Vereinigung, 59 (1957), 131-140.

6. G. Bastin, J.-M. Coron, Stability and boundary stabilization of 1-D hyperbolic systems, Birkhäuser/Springer, Progress in Nonlinear Differential Equations and their Applications, Subseries in Control, 88 (2016). 
7. J.-L. Lions, Y. MAdAY, G. TURINICI, Resolution d'EDP par un schema en temps 'parareel', Comptes Rendus de l'Academie des Sciences, Series I. Mathematics, 332(7) (2001), 661-668.

8. M. J. Gander, S. VAndeWAlle, Analysis of the parareal time-parallel timeintegration method, SIAM Journal on Scientific Computing, 29(2) (2007), 556-578.

9. S. Ulbrich, Generalized SQP methods with 'parareal' time-domain decomposition for time-dependent PDE-constrained optimization, SIAM, Real-time PDEconstrained optimization,Computational Science \& Engineering, 3 (2007), 145-168.

10. Gander, Martin J., Analysis of the parareal algorithm applied to hyperbolic problems using characteristics, Boletin de la Sociedad Espanola de Matematica Aplicada, 42 (2008), 21-35.

11. M. Heinkenschloss, A time-domain decomposition iterative method for the solution of distributed linear quadratic optimal control problems, Journal of Computational and Applied Mathematics, 173(1) (2005), 169-198.

12. W. SCHMAEDEKE, Mathematical theory of optimal control for semilinear hyperbolic systems in two independent variables, SIAM Journal on Control, 5 (1967), 138-153.

13. G. Leugering, J. P. G. Schmidt, On the modelling and stabilization of flows in networks of open canals, SIAM Journal on Control and Optimization, 41(1) (2002), 164-180.

14. G. Leugering, C. Rodriguez, Boundary feedback stabilization for the intrinsic geometrically exact beam model, https://arxiv.org/abs/1912.02543, (2020).

15. G. Leugering, A. Martin, M. Schmidt, M. Sirvent, Nonoverlapping domain decomposition for optimal control problems governed by semilinear models for gas flow in networks, Control and Cybernetics, 46(3) (2017), 191-225.

16. F. M. Hante, G. Leugering, A. Martin, L. Schewe, M. Schmidt, Challenges in Optimal Control Problems for Gas and Fluid Flow in Networks of Pipes and Canals: From Modeling to Industrial Applications, Industrial Mathematics and Complex Systems: Emerging Mathematical Models, Methods and Algorithms, Manchanda, Pammy and Lozi, Rene and Siddiqi, Abul Hasan, Eds., Industrial and Applied Mathematics, (2017), 77-122.

17. M. J. Gander, F. Kwok, J. SAlomon, ParaOpt: a parareal algorithm for optimality systems, SIAM Journal on Scientific Computing, 42(5) (2020), A2773-A2802.

18. M. J. GANDER, F. KWOK, Schwarz methods for the time-parallel solution of parabolic control problems, Domain decomposition methods in science and engineering XXII, Lecture Notes in Computational Science and Engineering, Springer, Cham, 104 (2016), 207-216.

19. Y. Maday, M.-K. Riahi, J. Salomon, Parareal in time intermediate targets methods for optimal control problems, Control and optimization with PDE constraints, Internat. Ser. Numer. Math., 164, 79-92, Birkhäuser/Springer Basel AG, Basel, 2013.

20. A. T. BARKer, M. Stoll, Domain decomposition in time for PDE-constrained optimization, Computer Physics Communications, 197 (2015), 136-143.

21. Wu, Shu-Lin And Huang, Ting-Zhu, A fast second-order parareal solver for fractional optimal control problems, Journal of Vibration and Control, 24(15) (2018), 3418-3433.

22. S.-L. WU, J. LIU, A parallel-in-time block-circulant preconditioner for optimal control of wave equations, SIAM Journal on Scientific Computing, 42(3) (2020), A1510-A1540. 
23. Y. Maday, J. Salomon, G. Turinici, Monotonic time-discretized schemes in quantum control, Numerische Mathematik, 103(2) (2006), 323-338.

24. Y. Maday, G. Turinici, A parareal in time procedure for the control of partial differential equations, Comptes Rendus Mathématique. Académie des Sciences, Paris, 335(4) (2002), 387-392.

25. P.-L. LiOns, On the Schwarz alternating method. III. A variant for nonoverlapping subdomains, Third International Symposium on Domain Decomposition Methods for Partial Differential Equations (Houston, TX, 1989), 202-223, SIAM, Philadelphia, PA, 1989.

26. R. Glowinski, P. LE TAllec, Augmented Lagrangian interpretation of the nonoverlapping Schwarz alternating method, Third International Symposium on Domain Decomposition Methods for Partial Differential Equations (Houston,TX, 1989), 224-231, SIAM, Philadelphia, PA, 1990.

27. H. BiALY, Iterative Behandlung linearer Funktionalgleichungen, Archive for Rational Mechanics and Analysis, 4 (1959), 166-176.

28. M. J. Gander, L. Halpern, F. NAtAF, Domain decomposition methods for wave propagation, Mathematical and numerical aspects of wave propagation (Santiago de Compostela, 2000), SIAM, Philadelphia, PA, (2000), 807-811.

29. F.-C. Отто, G. LuBe, A posteriori estimates for a non-overlapping domain decomposition method, Computing, 62 (1) (1999), 27-43.

30. S. E. Mikhailov, Traces, extensions and co-normal derivatives for elliptic systems on Lipschitz domains, J. Math. Anal. Appl., 1 (2011), 324-342.

31. R. Krug, G. Leugering, A. Martin, M. Schmidt, D. Weninger, TimeDomain Decomposition for Optimal Control Problems Governed by Semilinear Hyperbolic Systems with Mixed Two-Point Boundary Conditions (submitted), Friedrich-Alexander-Universität Erlangen-Nürnberg, 20, (2021).

32. R. Krug, G. Leugering, A. Martin, M. Schmidt, D. Weninger, TimeDomain Decomposition for Optimal Control Problems Governed by Semilinear Hyperbolic Systems (to appear in SICON), Friedrich-Alexander-Universität Erlangen-Nürnberg, 28, (2021).

33. Z. OPIAL, Weak convergence of the sequence of successive approximations for nonexpansive mappings, Bull. Amer. Math. Soc., 73, (1967), 591-597.

34. C. Corduneanu, Integral equations and applications, Cambridge University Press, Cambridge, (1991).

35. D. L. Russell, Optimal regulation of linear symmetric hyperbolic systems with finite dimensional controls, SIAM J. Control, 4, (1966), 276-294. 